Proceedings

\title{
Mitogenomics and Phylogeny of Seven African Saturniidae (Lepidoptera) ${ }^{\dagger}$
}

\author{
Rieze Straeuli ${ }^{1 *}$ and Barbara van Asch ${ }^{1}$
}

Citation: Straeuli, R.; van Asch, B. Mitogenomics and Phylogeny of Seven African Saturniidae (Lepidoptera), in Proceedings of the 1st International Electronic Conference on Entomology, 1-15 July 2021, MDPI: Basel, Switzerland, doi:10.3390/IECE10372

\section{Published: 30 June 2021}

Publisher's Note: MDPI stays neutral with regard to jurisdictional claims in published maps and institutional affiliations.

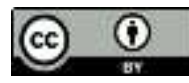

Copyright: (c) 2021 by the authors. Submitted for possible open access publication under the terms and conditions of the Creative Commons Attribution (CC BY) license (http://creativecommons.org/licenses /by/4.0/).
1 Department of Genetics, Stellenbosch University, Private Bag X1, Matieland 7602, South Africa; 24656879@sun.ac.za; bva@sun.ac.za

* Correspondence: 24656879@sun.ac.za

+ Presented at the 1st International Electronic Conference on Entomology (IECE 2021), 1-15 July 2021; Available online: https://iece.sciforum.net/.

\begin{abstract}
African Saturniidae (Lepidoptera) have not been included in recent mitochondrial phylogenies except for two edible species of economic importance in Southern Africa. To bridge this gap, we sequenced the complete mitochondrial genomes for seven additional species (Epiphora bauhinia, Heniocha dyops, Gonimbrasia tyrrhea, Bunaea alcinoe, Nudaurelia cytherea, Vegetia ducalis and Vegetia grimmia), including the first representatives of the tribe Micragonini for comparative mitogenomics and phylogenetic reconstruction of the family. The mitochondrial gene content and organization were conserved across Saturniidae. After testing for different datasets of genes, the only tree able to successfully recover the four tribes as monophyletic clades included the two rRNA gene sequences (12S rRNA and 16S rRNA) in addition to the full complement of 13 protein-coding genes.
\end{abstract}

Keywords: Monophyletic clades; ribosomal gene; Micragonini; tribes

\section{Introduction}

The family Saturniidae (emperor moths) includes 3,454 species distributed worldwide in a diversity of terrestrial habitats [1]. African emperor moths are understudied despite their ecological and economic relevance, as the caterpillars of many species are utilized for human consumption [2]. For example, mopane worms, the vernacular name for the caterpillars of Gonimbrasia belina and other Saturniidae in South Africa, are widely harvested in Southern Africa due to their high nutrient content [3]. Increased demands for favoured edible Saturniidae species, habitat destruction and unregulated harvesting are placing wild populations in serious danger of decline [4]. Currently, most research on the genetic diversity of Saturniidae has focused on species farmed in Asia for silk production [5]. African Saturniidae have remained largely unsurveyed, and mitochondrial phylogenies of the family have so far included only G. belina and Gynanisa maja, two of the most exploited edible caterpillars in Southern Africa [6]. This worked aimed to generated baseline genetic data on African Saturniidae, and to assess the phylogenetic position of seven species in four tribes: Heniocha dyops, Gonimbrasia tyrrhea, Bunaea alcinoe, Nudaurelia cytherea (Bunaeini), Epiphora bauhinia (Attacini), Vegetia ducalis and Vegetia grimmia (Micragonini). For that purpose, we sequenced and described the complete mitogenome of one individual of each species, and inferred their phylogenetic relationships with other Saturniidae.

\section{Materials and Methods}

\subsection{Sample Collection and DNA Extraction}


Caterpillars and adult specimens representative of the seven species were collected in Namibia and South Africa between 15 March 2018 and 12 March 2020 (Table 1). Adult specimens were stored at $-20^{\circ} \mathrm{C}$, and caterpillars were stored in $100 \%$ ethanol at room temperature until further analyses. DNA from the legs of caterpillars and adult specimens was extracted using a standard phenol-chloroform protocol.

Table 1. Collection data for seven species of African Saturniidae used for the recovery of complete mitogenomes, comparative mitogenomics and phylogenetic reconstruction within the family.

\begin{tabular}{llll}
\hline Species & Tribe & Region & Country \\
\hline Bunaea alcinoe & Bunaeini & Gauteng & South Africa \\
Epiphora bauhinia & Attacini & Okonjima & Namibia \\
Gonimbrasia tyrrhea & Bunaeini & Western Cape & South Africa \\
Heniocha dyops & Bunaeini & Windhoek & Namibia \\
Nudaurelia cytherea & Bunaeini & Western Cape & South Africa \\
Vegetia ducalis & Micragonini & Western Cape & South Africa \\
Vegetia grimmia & Micragonini & Western Cape & South Africa \\
\hline
\end{tabular}

\subsection{Morphological and DNA-Based Species Identification}

Morphological identification of specimens was performed following the available literature and taxonomic keys $[7,8]$. PCR amplifications of the standard COI barcoding region were performed in a total volume of $5 \mu \mathrm{L}$ containing $2.5 \mu \mathrm{L}$ of Qiagen Multiplex PCR Kit (QIAGEN), $0.5 \mu \mathrm{L}$ of each primer, $0.5 \mu \mathrm{L}$ of Milli-Q water and $1 \mu \mathrm{L}$ of template DNA. Thermocycling conditions consisted of initial denaturation at $95^{\circ} \mathrm{C}$ for $15 \mathrm{~min}$; 35 cycles of $94^{\circ} \mathrm{C}$ for $30 \mathrm{~s}, 50^{\circ} \mathrm{C}$ for $90 \mathrm{~s}$ and a final extension at $72^{\circ} \mathrm{C}$ for $10 \mathrm{~min}$. PCR products are sequenced unidirectionally using the BigDye Terminator v3.1 Cycle Sequencing Kit (Applied Biosystems), at the Central Analytical Facilities of Stellenbosch University, South Africa. Sequences are manually edited and trimmed on Geneious Prime v2020.2.2 and aligned using MAFFT algorithm plug-in [9] on Geneious Prime.

\subsection{Next-Generation Sequencing for Recovery of Mitochondrial Genomes}

DNA from one representative of each species was individually sequenced using the Ion Torrent Proton ${ }^{\mathrm{TM}}$ sequencing platform (ThermoFisher Scientific) available at the Central Analytical Facility of Stellenbosch University. Libraries were prepared using Ion Express $^{\mathrm{TM}}$ and gDNA Fragment Library (ThermoFisher Scientific, Waltham, MA, USA) according to the MAN0009847 REV J.0 protocol. The libraries were sequenced using Ion $540^{\mathrm{TM}}$ Chef sequencing solution kit. Massive parallel sequencing was performed on Ion Torrent S5 ${ }^{\mathrm{TM}}$ system using sequencing solutions, reagents and supplies according to manufacturer's protocol.

\subsection{Mitogenome Mapping, Assembly, and Annotations}

Reference-based assembly of the next-generation sequencing (NGS) reads was performed using complete mitogenomes available on GenBank. The closest relative for each species was selected based on COI sequence similarity estimated from the DNA barcodes used for confirming morphological identification of the specimens (data not shown). Mapping and assembly of the NGS reads was performed using the "Map to reference" function available on Geneious Prime under the low to medium sensitivity option and fine tuning up to five iterations. Open reading frames for the typical 13 metazoan mitochondrial protein coding genes (PCGs) were identified with Geneious Prime using the invertebrate mitochondrial genetic code. Transfer RNA (tRNA) genes were identified with ARWEN 
software (http://130.235.244.92/ARWEN/) using the default composite metazoan mitochondrial genetic code. The two ribosomal RNA (rRNA) genes and the non-coding ATrich region were annotated by comparison to other complete Saturniidae mitogenomes available on GenBank.

\subsection{Comparative Mitogenomics}

Nucleotide composition and AT- and GC-skews were calculated using Geneious Prime $[$ AT-skew $=(\mathrm{A}-\mathrm{T}) /(\mathrm{A}+\mathrm{T})$; GC-skew $=(\mathrm{G}-\mathrm{C}) /(\mathrm{G}+\mathrm{C})]$. Gene overlapping regions and intergenic spaces were counted manually. Relative synonymous codon usage was calculated in MEGA X [10] with invertebrate mitochondrial genetic code.

\subsection{Phylogenetic Analyses}

The phylogenetic reconstruction of the family Saturniidae included the new seven mitogenomes and all other sequences available on GenBank as of November 2020. The final dataset included 36 sequences across 25 species. Bombyx mori and Bombyx mandarina (Lepidoptera: Bombycidae) were used as outgroups. Phylogenetic analyses were performed separately on three datasets: (1) PCG123 - all PCGs and all codon positions, (2) PCG12 all PCGs with $3^{\text {rd }}$ codon position removed; and (3) PCG123+rRNA - all PCGs and the two rRNAs. Individual PCGs were extracted and stop codons were removed, and translation alignments were performed separately for each PCG using MAFFT in Genious Prime. Concatenated alignments were individually edited using GBlocks v0.91 (https://ngphylogeny.fr/tools/tool/276/form) for removing gaps and poorly aligned regions. The final alignments for the three datasets were partitioned using PartitionFinder2 on XSEDE [11] on the CIPRES Portal ver. 3.3 [12] (https://www.phylo.org/), using default settings. Bayesian Inference (BI) analyses were performed using the software MrBayes ver.3.2.6 [13] available on CIPRES. BI was conducted as follows: two independent runs of four heat chains, ten million generations run simultaneously, resampling ever 1,000 generations, first $25 \%$ of trees discarded as burn-in and the decision criterion for the convergence of the two runs set as an average split frequency of $\leq 0.01$. Confidence values for BI trees were calculated using Bayesian posterior probabilities (BPP). All trees were drawn using FigTree v1.4.3. [14].

\section{Results}

\subsection{Comparative Mitogenomics of Saturniidae}

\subsubsection{Genome Organization}

The complete mitogenomes of the newly sequenced African Saturniidae species were identical in gene content and organization, with 37 genes and an AT-rich non-coding region, and total size from $15,363 \mathrm{bp}$ (N. cytherea) to $15,218 \mathrm{bp}$ ( $V$. ducalis). Gene order was conserved across all species, with nine PCGs and 14 tRNAs located on the major strand (J) strand and four PCGs, eight tRNAs and two rRNAs located on the minor (N) strand. The non-coding AT- rich region, located between 12s rRNA and tRNA ${ }^{\text {Met }}$ ranged from 337 to $377 \mathrm{bp}$. The mitogenomes of Saturniidae have one difference compared to the hypothetical ancestral Arthropoda, located in the tRNA arrangenment M-I-Q (Figure 1) [15]. The new mitogenomes are biased towards $\mathrm{A}$ and $\mathrm{T}$ with the $\mathrm{A}+\mathrm{T}$ nucleotide composition ranging from $79.1 \%$ to $80.7 \%$, as typical is the case in insects. 
Figure 1. Linear map of the complete mitochondrial genomes of Epiphora bauhinia, Heniocha dyops, Gonimbrasia tyrrhea, Bunaea alcinoe, Nudaurelia cytherea, Vegetia ducalis and Vegetia grimmia (Lepidoptera: Saturniidae).

\subsubsection{Mitochondrial Protein-Coding Genes}

PCGs represent $73 \%$ of the total mitogenomes with $\mathrm{A}+\mathrm{T}$ contents ranging from $77.9 \%$ to $79.8 \%$ (Table 3 ). The gene with highest average $\mathrm{A}+\mathrm{T}$ content across all species was ATP8 (92\%). The A+T skew of the major strand was negative for all regions except ND1, ND4, ND4L and ND5, which had a slightly positive AT-skew in all species. Additionally, all PCGs for all species in this study had a slightly negative GC- skew. All PCGs were initiated by ATN start codons (ATA, ATT, ATG and ATC), except COX1 which is initiated by CGA. All the mitogenomes in this study follow the same trend, with the most frequently used codons being UUA (Leu), AUU (Ile) and AUA (Met). These codons have a high A/T content rather than $\mathrm{G} / \mathrm{C}$, and this reflects the high $\mathrm{A} / \mathrm{T}$ composition in the PCGs and mitochondrial genome of insects [16].

Table 2. Comparison of AT and GC-skews of seven African Saturniidae species.

\begin{tabular}{llllllllll}
\hline Species & $\mathbf{A} \%$ & $\mathbf{C} \%$ & $\mathbf{G} \%$ & $\mathbf{T} \%$ & $\mathbf{A}+\mathbf{T} \%$ & $\mathbf{G}+\mathbf{C} \%$ & AT-skew & GC-skew & Size (bp) \\
\hline Bunaea alcinoe & 40.20 & 12.40 & 6.90 & 40.20 & 80.40 & 19.30 & -0.03 & -0.29 & 15,305 \\
Epiphora bauhinia & 39.50 & 12.00 & 7.90 & 40.50 & 80.00 & 19.90 & -0.01 & -0.21 & 15,259 \\
Gonimbrasia tyrrhea & 39.60 & 12.30 & 7.30 & 40.70 & 80.30 & 19.60 & -0.01 & -0.26 & 15,299 \\
Heniocha dyops & 38.60 & 12.90 & 7.80 & 40.50 & 79.10 & 20.80 & -0.02 & -0.25 & 15,306 \\
Nudaurelia cytherea & 39.00 & 11.50 & 7.80 & 41.50 & 80.50 & 19.30 & -0.01 & 0.20 & 15,224 \\
Vegetia ducallis & 39.00 & 11.50 & 7.80 & 41.50 & 80.50 & 19.30 & -0.01 & 0.20 & 15,224 \\
Vegetia grimmia & 38.90 & 11.70 & 7.80 & 41.40 & 80.30 & 19.50 & -0.01 & 0.20 & 15,253 \\
\hline
\end{tabular}

\subsection{Phylogenetic Position of African Saturniidae within the Family}

Mitochondrial genomes are important genomic resources for reconstructing phylogenetic relationships among species [17]. To date, the only African Saturniidae mitochondrial genomes available on GenBank are those of G. belina and Gy.maja, and the family has been mostly represented by Asian species [18]. Our phylogenetic reconstructions (Figure 2A and $\mathrm{B}$ ) included all mitogenomes currently available for the family, as well as the seven additional sequences generated in this study. The PCG123 tree and PCG12 tree recovered identical topologies. Heniocha dyops, B. alcinoe, N. cytherea, and G. tyrrhea were part of the monophyletic tribe Bunaeini $(\mathrm{BPP}=1)$. The mitogenomes of $V$. ducalis and $V$. grimmia are the first available for the tribe Micragonini, which was monophyletic $(\mathrm{BBP}=1)$. Both PCG123 and PCG12 trees were unable to recover the tribal structure of Saturniini (Figure $2 \mathrm{~A}$ and B), as Saturniini was split into three groups, of which two were monophyletic and $N$. haraldi as a diverged branch. Previous studies showed the non-monophyly Saturniini and incongruent position of $N$. haraldi, however the splitting of Saturniini into two monophyletic clusters and $N$. haraldi separate from these two clusters is unexpected [19]. Two species were also recovered as non-monophyletic (Saturnia boisduvalii and Samia ricini). The PCG123+rRNA tree recovered the same non-monophyletic species clustering seen in the identical PCG123 and PCG12 trees, which also noted in previous studies $[5,6,18-24]$ but all four tribes were monophyletic. it is the most successful phylogenetic 
reconstruction of the family Saturniidae produced by this study. These results therefore concur with the basal division of the subfamily into the Holarctic and Oriental tribes Bunaeini, Urotini and Saturniinae, and the Afrotopical tribes Micragonini [5,17]. PCG123+rRNAs is the only phylogeny that recovered all four tribes as monophyletic clades (Figure 3).

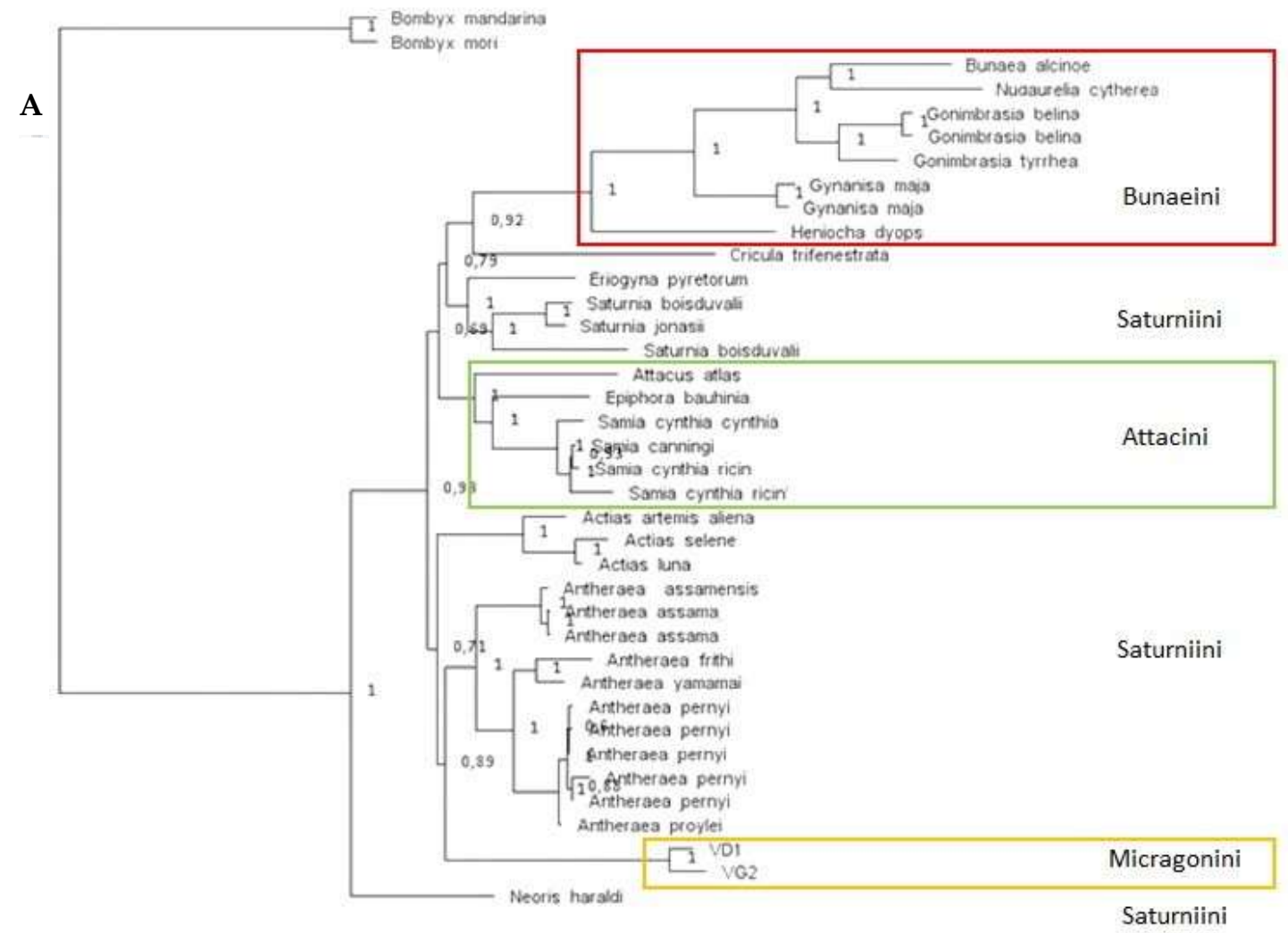




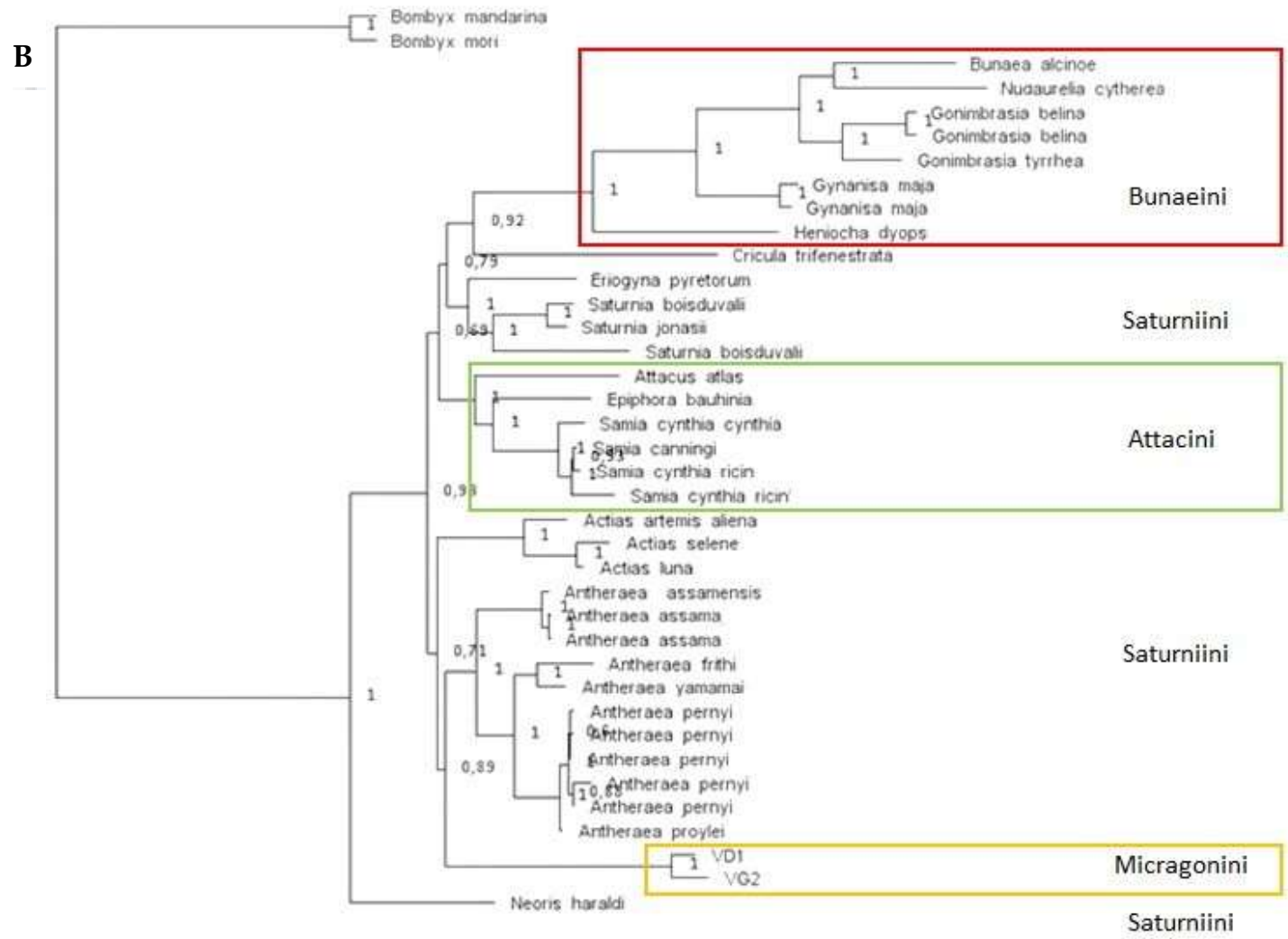

Figure 2. Bayesian reconstruction of the mitochondrial phylogeney of Saturniidae based on mitochondrial protein-coding genes (PCG). Nodal statistical support is given Bayesian posterior probability. (A) PCG12 (1st and 2nd codon positions). (B) PCG123 (all codon positions). 


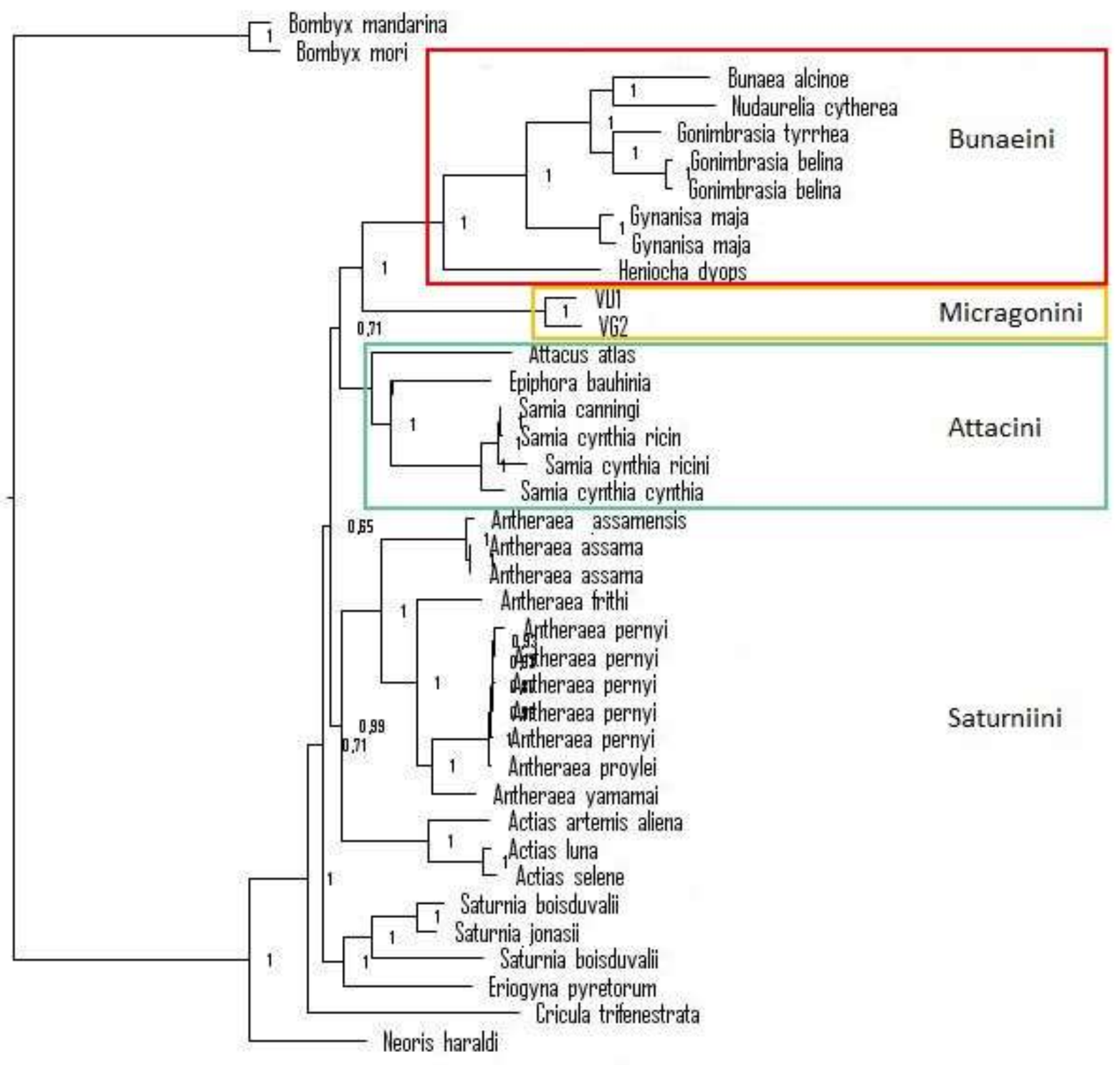

Figure 3. Bayesian reconstruction of the mitochondrial phylogeny of Saturniidae using all 3-codon position of the total compliment of 13 mitochondrial protein coding genes and two rRNA genes (PCG123+rRNA). Nodal statistical support is given Bayesian posterior probability.

\section{Conclusion}

The addition of seven mitogenomes assisted in clarifying the mitochondrial phylogenetic relationships of Saturniidae and showed that the current tribal structure can only be recovered by using the total complement of PCGs and rRNAs genes for the analyses. Our contribution to genetic baseline information on Africa Saturniidae is significant, as most of the data currently available is for Asian silk moths.

Supplementary Materials: No supplementary material is included in this paper. 
Author Contributions: BVA conceptualized the study and RS performed laboratory work and data analyses. All authors contributed to the writing, reviewing and editing of the manuscript and agreed to the final version for publication.

Funding: This research was funded by National Research Foundation of South Africa (NRF).

Informed Consent Statement: Not applicable.

Data Availability Statement: The sequences generated in this study are not yet publicly available.

Acknowledgements: The authors are grateful to William Versfeld, Gail Morland, Dylan Dyer and Chrissie Fourie for their assistance with sample collection.

Conflicts of Interest: The authors declare no conflict of interest.

\section{References}

1. Kitching, I. J. A global checklist of the Bombycoidea (Insecta: Lepidoptera). Biodivers. Data J 2018, 6, 1-12.

2. Van Huis, A. Edible insects are the future? Proc. Nutr. Soc 2016,75, 294-305.

3. Illgner, P.; Nel, E.; G. and Dec, N. The Geography of Edible Insects in Sub-Saharan Africa : A Study of the Mopane Caterpillar: The Geography of Edible Insects in Sub-Saharan Africa : a study of the Mopane Caterpillar. The, S., Journal 2016, 166, 336351.

4. Thomas, B. Sustainable harvesting and trading of mopane worms (Imbrasia belina) in Northern Namibia: An experience from the Uukwaluudhi area. Int. J. Environ. Stud 2013,70, 494-502.

5. Regier, J. C. Phylogenetic relationships of wild silkmoths (Lepidoptera: Saturniidae) inferred from four protein-coding nuclear genes. Syst. Entomol 2008, 33, 219-228.

6. Langley, J.; Van der Westhuizen, S., Morland, G. \& van Asch, B. Mitochondrial genomes and polymorphic regions of Gonimbrasia belina and Gynanisa maja (Lepidoptera: Saturniidae), two important edible caterpillars of Southern Africa. Int. J. Biol. Macromol 2020, 144, 632-642.

7. Cooper M, C. M. The emperor moths of KwaZulu-Natal.New Germany: Perinoceras Press, 2002; pp 100-121.

8. Pinhey, E. C. G. Emperor moths of South and South Central Africa. C Struik (Pty) Ltd: Cape Town, 1972.

9. Katoh, K. and Standley, D. M. Article Fast Track MAFFT Multiple Sequence Alignment Software Version 7: Improvements in Performance and Usability. doi:10.1093/molbev/mst010.

10. Kumar, S.; Stecher, G.; Li, M.; Knyaz, C. and Tamura, K. MEGA X : Molecular Evolutionary Genetics Analysis across Computing Platforms 2018, 35, 1547-1549.

11. Lanfear, R.; Frandsen, P. B.; Wright, A. M.; Senfeld, T. and Calcott, B. PartitionFinder 2: New Methods for Selecting Partitioned Models of Evolution for Molecular and Morphological Phylogenetic Analyses. Mol. Biol. Evol 2016, 34, 772-773.

12. Miller, M. A.; Pfeiffer, W. and Schwartz, T. Creating the CIPRES Science Gateway for inference of large phylogenetic trees. 2010 Gatew. Comput. Environ. Work. GCE 20102010 doi:10.1109/GCE.2010.5676129.

13. Huelsenbeck, J. P. and Ronquist, F. MRBAYES: Bayesian inference of phylogenetic trees. Bioinformatics 2001, 17, 754-755.

14. Rambaut, A. FigTree ver1.4.3. http://tree.bio.ed.ac.uk/software/figtree/ 2007.

15. Boore, J. L. Animal mitochondrial genomes. Int. J. Environ. Stud 1999,27, 1767-1780.

16. Bao, L.; Zhang, Y.; Gu, X.; Gao, Y. and Yu, Y. Genomics The complete mitochondrial genome of Eterusia aedea ( Lepidoptera , Zygaenidae ) and comparison with other zygaenid moths. Genomics 2019, 111, 1043-1052.

17. Cameron, S. L. Insect Mitochondrial Genomics: Implications for Evolution and Phylogeny. Annu. Rev. Entomol 2014, 59, 95117. 
18. Friedlander, T. P,; Peigler, R. S. and Regier, J.C. Monophyly, composition, and relationships within Saturniinae (Lepidoptera: Saturniidae): Evidence from two nuclear genes. Insect Syst. Evol 2002, 33, 9-21.

19. He, Y.; Wang, X. and Chen, L. S. The complete mitochondrial genome of Neoris haraldi Schawerda (Lepidoptera: Saturniidae). Mitochondrial DNA Part B Resour 2017, 2, 625-626.

20. Brahma, D.; Neli, S.; Saikia, P. and Dutta, S. Morphological and productivity differences between Samia Ricini, Samia Canningi and their cross. The Ecoscan 2011, 1, 287-290.

21. Friedlander, T. P. Two Nuclear Genes Yield Concordant Relationships within Attacini (Lepidoptera: Saturniidae). Mol. Phylogenet. Evol 1998, 9, 131-140.

22. Chen, M. Complete mitochondrial genome of the atlas moth, Attacus atlas (Lepidoptera: Saturniidae) and the phylogenetic relationship of Saturniidae species. Gene 2014, 545, 95-101.

23. Singh, D. The mitochondrial genome of Muga silkworm (Antheraea assamensis) and its comparative analysis with other lepidopteran insects. PLoS One 2017, 12, 1-23.

24. Sima, Y. H. The complete mitochondrial genome of the Ailanthus silkmoth, Samia cynthia cynthia (Lepidoptera: Saturniidae). Gene 2013, 526, 309-317. 